\title{
Assessing socioeconomic vulnerability to dengue fever in Cali, Colombia: statistical vs expert-based modeling
}

Michael Hagenlocher ${ }^{1 *}$, Eric Delmelle ${ }^{2}$, Irene Casas $^{3}$ and Stefan Kienberger ${ }^{1}$

\begin{abstract}
Background: As a result of changes in climatic conditions and greater resistance to insecticides, many regions across the globe, including Colombia, have been facing a resurgence of vector-borne diseases, and dengue fever in particular. Timely information on both (1) the spatial distribution of the disease, and (2) prevailing vulnerabilities of the population are needed to adequately plan targeted preventive intervention. We propose a methodology for the spatial assessment of current socioeconomic vulnerabilities to dengue fever in Cali, a tropical urban environment of Colombia.
\end{abstract}

Methods: Based on a set of socioeconomic and demographic indicators derived from census data and ancillary geospatial datasets, we develop a spatial approach for both expert-based and purely statistical-based modeling of current vulnerability levels across 340 neighborhoods of the city using a Geographic Information System (GIS). The results of both approaches are comparatively evaluated by means of spatial statistics. A web-based approach is proposed to facilitate the visualization and the dissemination of the output vulnerability index to the community.

Results: The statistical and the expert-based modeling approach exhibit a high concordance, globally, and spatially. The expert-based approach indicates a slightly higher vulnerability mean (0.53) and vulnerability median (0.56) across all neighborhoods, compared to the purely statistical approach (mean $=0.48$; median $=0.49$ ). Both approaches reveal that high values of vulnerability tend to cluster in the eastern, north-eastern, and western part of the city. These are poor neighborhoods with high percentages of young (i.e., $<15$ years) and illiterate residents, as well as a high proportion of individuals being either unemployed or doing housework.

Conclusions: Both modeling approaches reveal similar outputs, indicating that in the absence of local expertise, statistical approaches could be used, with caution. By decomposing identified vulnerability "hotspots" into their underlying factors, our approach provides valuable information on both (1) the location of neighborhoods, and (2) vulnerability factors that should be given priority in the context of targeted intervention strategies. The results support decision makers to allocate resources in a manner that may reduce existing susceptibilities and strengthen resilience, and thus help to reduce the burden of vector-borne diseases.

Keywords: Dengue fever, Vulnerability, Composite indicators, Expert-based vs. statistical modeling, GIS, Geovisualization, Vector-borne diseases, Colombia

\footnotetext{
* Correspondence: michael.hagenlocher@sbg.ac.at

${ }^{1}$ Interfaculty Department of Geoinformatics - Z_GIS, University of Salzburg,

Schillerstraße 30, Salzburg 5020, Austria

Full list of author information is available at the end of the article
} 


\section{Background}

Severe outbreaks of vector-borne diseases (VBDs) and their expansion pose a serious challenge to vulnerable populations. Recent changes in climatic conditions, greater resistance to insecticides and new public health policies have changed the dynamics of VBDs, such as dengue fever or malaria [1-3]. Projected changes in climate conditions [4] along with other factors, such as population growth, urbanization, lack of sanitation, and ineffective mosquito control are expected to result in a geographical expansion of dengue fever in the coming decades [5]. While malaria is still the most significant communicable disease $[2,6]$, dengue fever outbreaks have recently resurfaced $[7,8]$. Dengue fever is a vectorborne viral infection, transmitted among humans by the female Aedes aegypti mosquito [9]. It is prevalent in many tropical and sub-tropical regions across the globe [10-15]. Urban and suburban environments in those regions are particularly fragile due to rapid population movement (e.g. massive influx of migrants, causing unorganized urbanization) and the abundance of potential breeding sites.

In Colombia, South America, dengue fever reemerged in the 1970s after being eradicated in the 1950s and 1960s [16]. Ever since the disease has become endemic, presenting periodic outbreaks in 1991, 1994, 1998, 2001, 2006, and more recently in 2010. Most outbreaks have been of serotype 1 (DENV-1) and 2 (DENV-2) [17], however, in the last decade type 3 (DENV-3) and type 4 (DENV-4) have also been present [18]. Individuals infected with a particular serotype develop immunity to that type. When a serotype has not circulated for a while the population at risk of contracting the disease increases (in Colombia, this number currently equals 26 million individuals). These individuals are generally confined below 1,800 meters elevation, corresponding to nearly $80 \%$ of the total area of the country. Dengue fever in the city of Santiago de Cali (referred to as Cali from here on) has followed a similar temporal pattern to that of Colombia in general [18]. Based on the City's Health Municipality, significant dengue outbreaks occurred in 1995 with 6,433 cases reported, in $2002(\mathrm{n}=4,358), 2005$ $(\mathrm{n}=2,338)$ and $2010(\mathrm{n}=9,600)$ [17-19]. In 2010, the total number of cases was the highest in the last 25 years.

Three critical areas warranting efforts for reducing the burden of dengue fever are (1) identifying factors responsible for its distribution, (2) conducting proactive programs to reduce existing health vulnerabilities, and (3) strengthening existing capacities for creating more resilient societies on all levels (i.e., from global to local). To be effective, these programs must be based on up-to -date and reliable information on existing vulnerabilities and capacities on site, which is the scope of our paper.
Vulnerability is a well-established concept within the disaster risk reduction and the climate change adaptation communities [20-23]. As it helps to identify intervention options for reducing susceptibilities and strengthening resilience to VBDs independent of current disease prevalence, the emerging concept of vulnerability assessment holds promises in public health. To date, some studies have been published on vulnerability to VBDs in general [3,24-26], and dengue in particular [27-31]. A handful of those have addressed vulnerability to dengue fever in Latin America [27,28]. De Mattos Almeida et al. [27] analyzed the link between different risk categories and socioeconomic, demographic and urban-infrastructure characteristics in an urban area of Brazil, while Martinez et al. [28] used Geographic Information Systems (GIS) for mapping vulnerability to dengue in the City of Havana, Cuba. However, these studies lack a clear conceptual vulnerability framework, thus making the comparability and reproducibility of their results difficult. Most research integrating geospatial analysis for monitoring the dynamics of dengue fever has focused on developing disease surveillance systems [32], assessing exposure [33], or measuring determinants for dengue infection [34], while vulnerability is not integrated. Strategies which solely focus on vector control, reducing exposure or treatment of the disease may provide noticeable health benefits, but could be less effective at reducing the total health burden in the long term than integrative approaches aimed at underlying causes of vulnerability $[31,35]$. This is also supported by Jones and Williams [36], who advocate for an integrated approach to infectious disease control.

The objective of this paper is to perform a spatial assessment and evaluation of relative levels of socioeconomic vulnerability between different neighborhoods in Cali, Colombia independent of dengue fever prevalence. In the wider context of dengue risk, the focus is on modeling vulnerability (as a component of risk), i.e. the predisposition of the population of Cali of being adversely affected by the disease.

Based on a holistic vulnerability framework (i.e., a deductive approach) we use both statistical and expertbased approaches for the analysis and aggregation of singular socioeconomic and demographic indicators, and compare the outcomes using geo-statistical methods.

\section{Materials and methods}

\section{Study area}

Cali's core urban area is located west of the Cauca River with the Farallones Mountains acting as a natural barrier to the further expansion of the city. Cali generally experiences two rainy seasons from April to July and September to December. Located in an elevation of approximately 1,000 meters above sea level, the city's 
average temperature is $26^{\circ} \mathrm{C}$ with an annual average precipitation of $1,000 \mathrm{~mm}$ covering most of the metropolitan area [37]. With an estimated population of 2.3 million, Cali is currently the third largest city in Colombia. The city is administratively organized in 22 communes, which are divided into 340 neighborhoods (Figure 1). Communes are neighborhood groupings based on homogeneous demographic and socioeconomic characteristics. Neighborhoods are stratified into six socioeconomic classes based on the type of housing, urban environment, and urban context, one being the lowest and six the highest. Peripheral neighborhoods are characterized by high-density, low-income population with unplanned urbanization, including squatter settlements along the river banks [38]. This has been exacerbated by the influx of migrants displaced by the armed conflict who can only afford to settle in areas where poor living conditions are present. Casas et al. [39] discuss the infrastructure of such regions, and its impact on health access. The poor infrastructure of these neighborhoods may result in open-air waste water channels, while several households make use of rainfall cisterns for drinking water [14]. Thus, ample sources of stagnant water (i.e., ideal mosquito-breeding habitats) are present around the city. Currently, health authorities in the city of Cali rely on a preventive dengue control strategy where they spray bacterial larvicides to stagnant water sources every two weeks.

\section{Vulnerability: conceptual framework}

Our study makes use of the risk and vulnerability framework [40] which was developed in the European research project MOVE (Methods for the Improvement of Vulnerability Assessment in Europe). While the MOVE framework was elaborated in the context of natural hazard research and adaptation to climate change, it is modified in this paper to guide risk and vulnerability assessments in the VBD domain. The framework provides a conceptualization of the complex and multi-dimensional nature of vulnerability of a society and its population at different spatial and temporal scales. The MOVE framework characterizes vulnerability through three key factors, namely (1) exposure - reflecting the extent to which a unit of assessment falls within the geographical range of a hazard event, (2) susceptibility (SUS) - which describes the predisposition of elements at risk to suffer harm, and (3) lack of resilience (LoR), which is determined by limitations in terms of access to, and mobilization of the resources of a community or social-ecological system in responding to a particular hazard. As the MOVE framework intends to

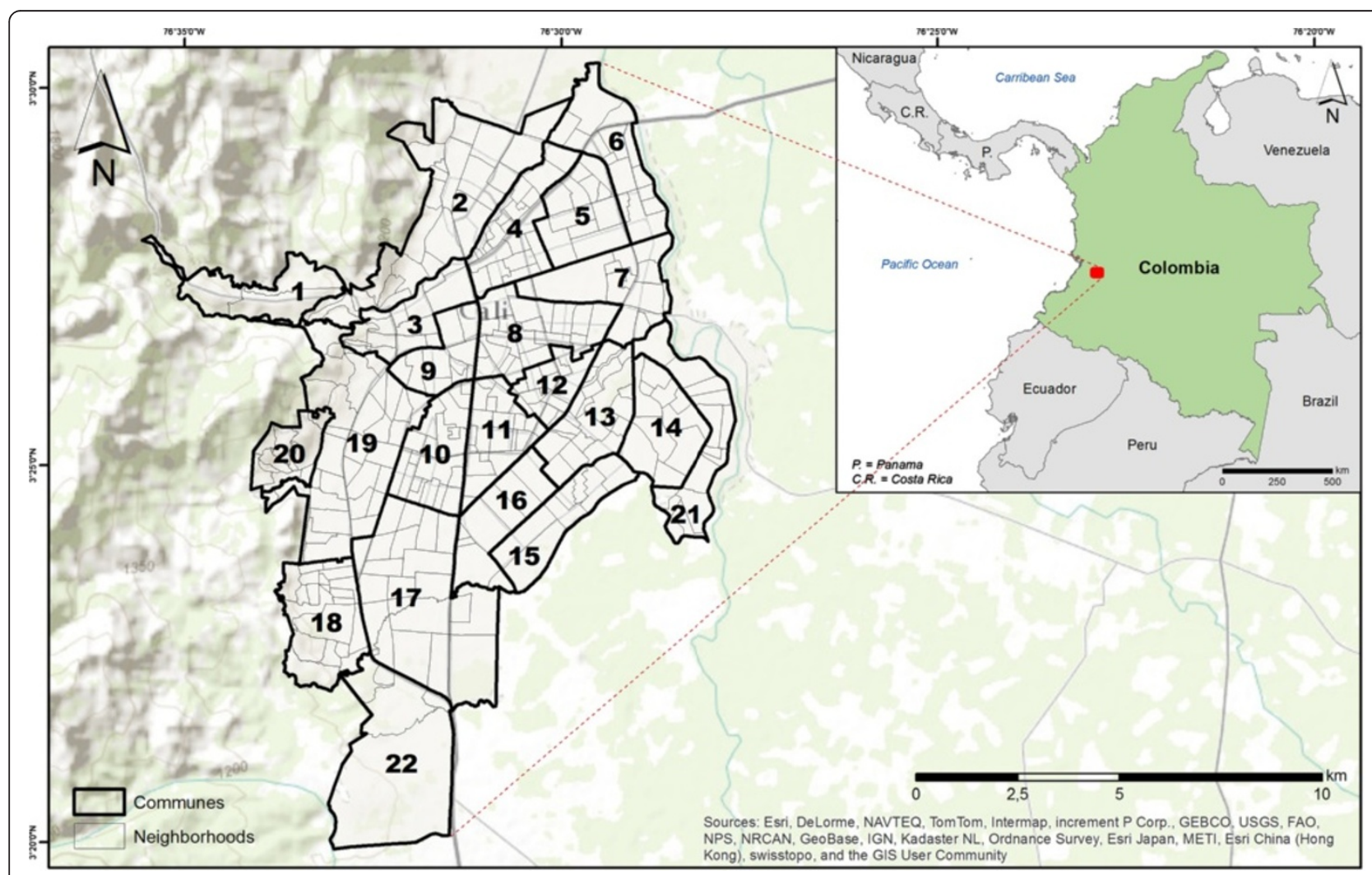

Figure 1 Base map showing the location of Cali, Colombia. 
describe risk from a holistic perspective, different thematic dimensions characterize vulnerability: social, economic, environmental, physical, cultural and institutional [40]. In this paper, we define vulnerability as the predisposition of a system and its population of being adversely affected by the disease. Population vulnerability is determined by their degree of susceptibility, as well as the individual's (lack of) resilience. In this conceptualization of vulnerability, the susceptibility (SUS) domain characterizes the predisposition of being negatively affected by an outbreak, whereas lack of resilience (LoR) includes (lacking) capacities to anticipate, cope or recover from the (burden of the) disease. In contradiction to the MOVE framework, we excluded the exposure component from the vulnerability analysis, as all neighborhoods have been affected by dengue outbreaks in recent years. We acknowledge exposure as part of the overall risk equation, where risk is defined by the location and magnitude of dengue occurrence, and the characteristics of exposed and vulnerable population groups. Since estimating dengue risk is not the focus of this paper, we assess socioeconomic vulnerability based on societal (i.e., the propensity for human well-being to be affected by disruption to individual and collective social systems and their characteristics) and economic (i.e., the propensity for loss of economic value from disruption of productive capacity) factors. By decomposing the complex, multi-dimensional phenomenon of vulnerability into its different components, the purpose of the framework is to serve as a guidance tool for the development of a representative set of indicators, suitable to represent socioeconomic vulnerability to dengue fever for the city of Cali, Colombia.

\section{Constructing a composite index of socioeconomic vulnerability}

In order to provide updated information on the multifaceted nature of prevailing vulnerabilities to dengue fever in Cali, Colombia, a composite vulnerability index was developed. The index builds on a set of underlying socioeconomic and demographic indicators. A multi-step and iterative workflow (Figure 2) is adopted following OECD [41] guidelines. Relevant stages include: (1) definition of the conceptual framework, (2) identification of a representative set of indicators based on existing literature, (3) data transformation, (4) analysis and imputation of missing values, (5) normalization, (6) multivariate analysis and establishment of final indicator set, (7) weighting, (8) aggregation and (9) visualization, as detailed in Malczewski [42].

According to [41], there are multiple options for composite indicator construction. As indicated in Figure 2, we aim to compare the results derived by expert-based and statistical modeling approaches for the development of a socioeconomic vulnerability index. The main difference between both approaches is the way in which indicator weights are derived: While for the purely statistical approach multivariate analysis is used to derive indicator weights, the latter are assessed making use of traditional budget allocation for the expert-based approach (Figure 2). The following paragraphs provide an overview of the individual stages in constructing the composite vulnerability index.

Drawing on the conceptual vulnerability framework, within stage 2, a preliminary set of 23 socioeconomic indicators (Table 1) was identified based on a systematic review of literature and available datasets. The choice

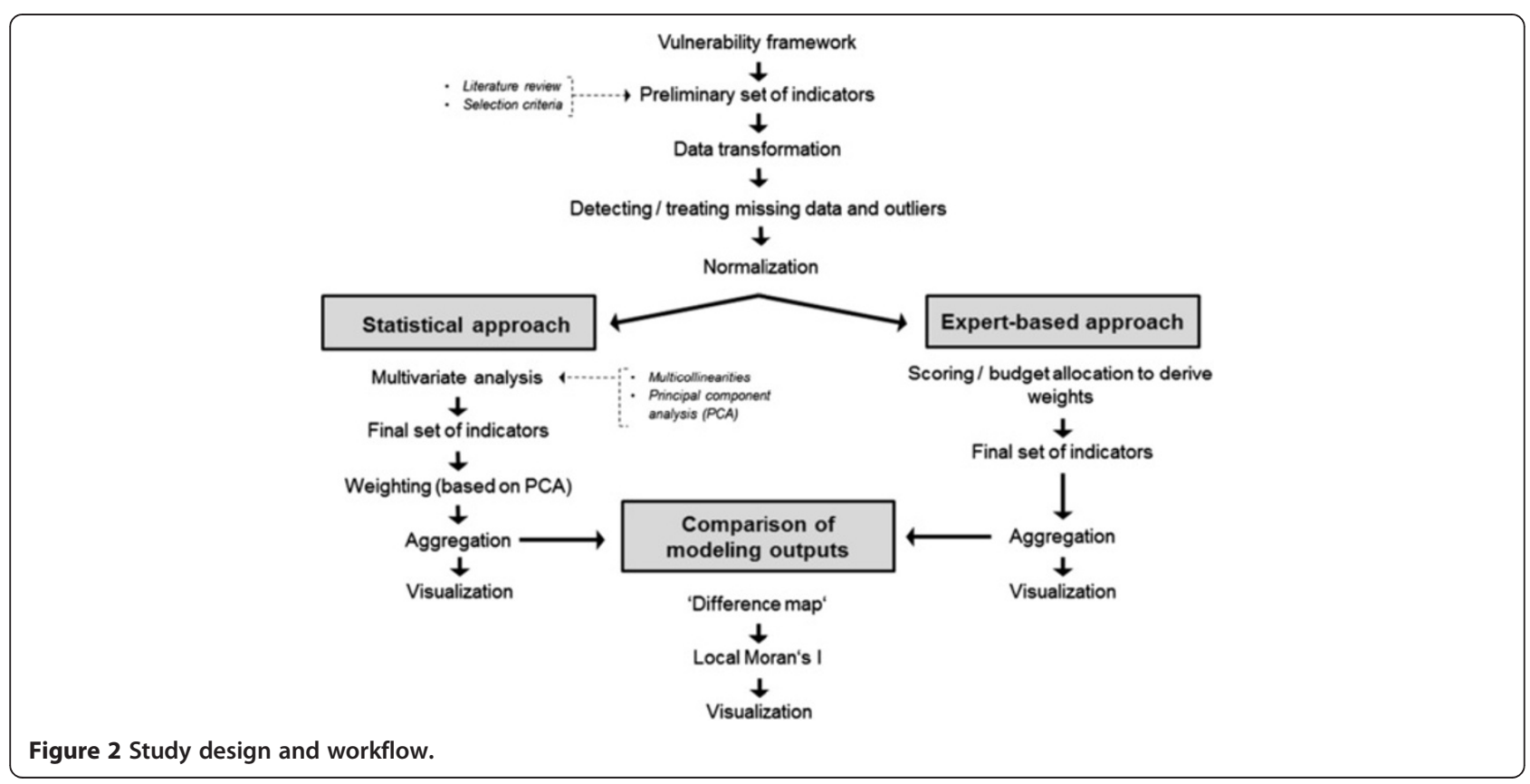


Table 1 Preliminary list of susceptibility (SUS) and lack of resilience (LoR) indicators ${ }^{\mathrm{a}}$

\begin{tabular}{llll}
\hline Domain & Indicator name & Domain & Indicator name \\
\hline SUS & Population density $\left(\mathrm{km}^{2}\right)$ & LoR & Households without a phone (\%) \\
SUS & Density of occupied households $\left(\mathrm{km}^{2}\right)$ & LoR & People who cannot read or write (\%) \\
SUS & Residents from age 0 to $4(\%)$ & LoR & People with no education (\%) \\
SUS & Residents from age 5 to $14(\%)$ & LoR & People - secondary/higher education level (\%) \\
SUS & Residents from age 15 to $29(\%)$ & LoR & Travel time to nearest hospital (min.) \\
SUS & Residents of age above 30 $(\%)$ & LoR & Distance to nearest hospital (m) \\
SUS & Black population (\%) & LoR & Mean hospital density (km²) \\
SUS & White population (\%) & LoR & Employed population (\%) \\
SUS & People with disabilities (\%) & LoR & Unemployed population (\%) \\
SUS & Households without water access (\%) & LoR & People doing housework (\%) \\
SUS & Households without sewer system (\%) & LoR & Retired people (\%) \\
SUS & Building stratification (1-6) & &
\end{tabular}

${ }^{\mathrm{a} B a s e d}$ on the outcomes of the literature survey and data availability (SUS = susceptibility; LoR = lack of resilience).

and selection of indicators and in particular socioeconomic factors, as outlined by Bates et al. [24,25], is a critical process in the overall method as it refers to evidence provided in scientific studies.

From literature population density, age groups, education levels, houses with different types of water access and the density of commercial areas have been identified as primary indicators in vulnerability assessments $[27,28,31]$. Despite dengue fever being regarded as a childhood disease [31], Guha-Sapir and Schimmer [43] observed that dengue hemorrhagic fever is also prevalent in older age groups. Variations of susceptibility levels in different age groups have also been observed in other studies $[3,28,31,44]$. Differences in race/ethnicity also have a marked impact on human susceptibility to dengue fever $[43,45,46]$. Sierra et al. [45], for example, observed that black individuals have a reduced risk for dengue fever compared to white/Caucasian individuals. Within the lack of resilience (LoR) domain, different levels of employment, and therefore access to financial resources, were identified as important coping and recovery indicators by Martinez et al. [28]. We included access to health facilities as an additional indicator, since adequate access to healthcare increases resilience (i.e., people's capacity to cope and to recover) and may reduce dengue mortality [31]. We integrated building stratification - as a proxy for poverty levels and housing conditions - in the LoR domain, as for example poor housing quality was found to increase susceptibility to the disease [31]. Poor housing conditions facilitate movement of vectors between interior and exterior, but are also associated with a lack of other infrastructure, increasing the overall susceptibility to dengue [31].

During the selection process, standard criteria for indicator selection such as validity, sensitivity, reproducibility and scale $[20,47]$ were accounted for. The indicators were associated with either the SUS or the LoR domain of vulnerability (Table 1 ).

The study builds on 2005 census data (i.e., the last census year) at the neighborhood level for the city of Cali which was made available by the municipality. It integrates ancillary geospatial datasets such as city roads, public transportation system network, and hospitals. The roads and public transportation network were obtained from various municipal agencies, while hospital addresses were acquired from the municipality and manually geocoded. This data was further processed using a GIS to calculate the average travel time between the centroid of each neighborhood and the closest hospital using public transportation [48] as well as the hospital density (in $\mathrm{km}^{2}$ ) in the city, by means of kernel density estimation. Finally, as vulnerability is a humancentered concept [49], all neighborhoods without permanent population (i.e., 'residents') were excluded from further analysis. This includes public parks, industrial areas, water treatment plants, military bases, cemeteries and academic institutions.

Within stage 3, raw data was transformed to achieve a better comparability of neighborhoods of different size and population or household counts [50]. For example, the absolute number of individuals who are not able to read or write was transformed into a relative measure. Following the same logic, areal density measures were computed in a GIS to transform the number of hospitals per neighborhood into a relative measure.

Descriptive statistics were used for each indicator within stage 4 to evaluate the degree of missing data and potential outliers. Following guidelines published by Groenefeld and Meeden [51], four indicators with skewness $>2$ and kurtosis $>3.5$ (i.e., hospital density; travel time to closest hospital; percentage of households without water; percentage of households without a phone) 
were highlighted as statistically 'problematic' with regard to potential outliers, and two indicators (i.e., building stratification; travel time to closest hospital) revealed a minimum percentage of missing data $(<2 \%)$. Missing values in the building stratification layer were imputed by integrating local expert-knowledge, while mean travel time to the closest hospital (in minutes) was imputed with the mean travel time of adjacent neighborhoods.

All datasets were standardized (stage 5) using linear min-max normalization (equation 1) and z-score standardization (equation 2), according to Malczewski [42].

$$
v_{i}^{\prime}=\frac{\left(v_{i}-v_{\min }\right)}{\left(v_{\max }-v_{\min }\right)} * \operatorname{sign}+0.5 *(1-\operatorname{sign})
$$

Where $v_{i}$ refers to the actual pixel value, $v_{\min }$ and $v_{\max }$ values derive from the original value range and sign (or polarity) indicates whether the indicator contributes positively or negatively to vulnerability. This results in normalized values $\left(v_{i}^{\prime}\right)$ in the zero to one interval. Minmax normalization was chosen, as z-score produces negative indicator values and thus complicates the final aggregation of the indicators. Z-score standardization was selected as an additional technique as it produces output datasets with a mean of zero and a variance of one, which is useful for subsequent statistical analysis (e.g. principal component analysis).

$$
v_{i}^{\prime \prime}=\frac{\left(v_{i}-\bar{v}\right)}{\sigma} * \operatorname{sign}
$$

with $\bar{v}$ corresponding to the mean and $\sigma$ the standard deviation of the data. $V_{i}$ " is the resulting standardized value.

To detect and ultimately reduce existing multicollinearities in the data, the correlation coefficient $(r)$, as well as variance inflation factors (VIF), were calculated within the SUS and LoR domain (stage 6). Based on thresholds published by the OECD [41], the following indicators (with $r>0.9$ and/or VIF $>5$ ) were excluded from further analysis: population density, proportion of white population, households without sewer system, people with no education, distance to closest hospital and proportion of population above 30 years.

For the statistical modeling approach, a principal component analysis (PCA) was conducted within each of the two domains to further test the robustness of the selected indicators (z-scores), and to derive indicator weights (as described below). Based on eigenvalues greater than 1.0 (Kaiser criterion), and a scree plot which shows a distinct break in the eigenvalues [41], the components which explain the majority of the variance among all neighborhoods were identified.

Weights were derived for the individual indicators (stage 7). Two different approaches were pursued to determine the weights to use (Figure 2). For the statistical approach, weights were calculated based on PCA and factor analysis [41]. For the expert-based approach, expert opinions were used to derive weights. Using a budget allocation approach, four local domain experts of differing backgrounds (epidemiologists and public health specialists from the Health Municipality of Cali) were asked to distribute a total of 100 points across the individual indicators within each of the two vulnerability domains (i.e., SUS, LoR).

The normalized indicators (min-max) were aggregated (stage 8) according to their respective domains (SUS, LoR):

$$
\text { SUS } / \text { LoR }=\frac{\sum_{i=1}^{n} w_{i} v_{i}^{\prime}}{n}
$$

where SUS/LoR refers to either of the two vulnerability domains for a given neighborhood, $n$ equals the number of indicators, $w_{i}$ represents the weights for indicator $i$ (either based on statistical or expert-based weighting; see Table 2) and $v_{i}^{\prime}$ is the normalized value (min-max) of indicator $i$.

The aggregation of the two domains (i.e., SUS and LoR) into the final composite indicator of socioeconomic vulnerability was then performed using the equation below, while taking into account specific weights for the two domains as detailed below.

$$
V U=\frac{\sum_{j=1}^{n} w_{j} X_{j}}{m}
$$

In the equation, $V U$ refers to the vulnerability index for a given neighborhood, $m$ equals the number of domains, $w_{j}$ represents the weights for domain $j$ and $X_{j}$ is the normalized value (min-max) of domain $j$ (i.e., SUS, LoR). For both the statistical and the expertbased approach, a weight of 0.47 (eight SUS indicators divided by 17 total indicators) was assigned to the SUS domain, while the LoR domain was assigned a weight of 0.53 (nine LoR indicators divided by 17 total indicators). This was done to achieve a balanced structure between both domains within the composite vulnerability index.

To ease the interpretation of the modeling results, the final expert-based and statistical vulnerability index values were normalized within the zero to one range, where zero reflects a very low and one a very high socioeconomic vulnerability to dengue fever.

\section{Comparative assessment of modeling approaches}

To assess differences in modeling outputs between the statistical and the expert-based approach, the difference $(\Delta)$ between both vulnerability indices was calculated (equation 5) and visualized in a GIS. Therefore, the normalized index values of the statistical approach $\left(V U_{\text {stat }}\right)$ 
Table 2 Final list of indicators, sign and weights (expert weights, statistical weights)

\begin{tabular}{|c|c|c|c|c|c|}
\hline Domain & Indicator name & $\operatorname{Sign}^{a}$ & Expert weights & Statistical weights & Data source \\
\hline SUS_01 & Density of occupied households $\left(\mathrm{km}^{2}\right)$ & + & 0.11 & 0.07 & Census 2005 \\
\hline SUS_02 & Residents from age 0 to 4 (\%) & + & 0.16 & 0.22 & Census 2005 \\
\hline SUS_03 & Residents from age 5 to $14(\%)$ & + & 0.14 & 0.23 & Census 2005 \\
\hline SUS_04 & Residents from age 15 to $29(\%)$ & + & 0.13 & 0.10 & Census 2005 \\
\hline SUS_05 & White population (\%) & + & 0.10 & 0.12 & Census 2005 \\
\hline SUS_06 & People with disabilities (\%) & + & 0.03 & 0.11 & Census 2005 \\
\hline SUS_07 & Building stratification (1-6) & - & 0.14 & 0.15 & Census 2005 \\
\hline \multirow[t]{2}{*}{ SUS_08 } & Households without water access (\%) & + & 0.19 & N/A & Census 2005 \\
\hline & & & Sum $=1$ & sum $=1$ & \\
\hline LoR_01 & Households without a phone (\%) & + & 0.03 & 0.17 & Census 2005 \\
\hline LoR_02 & People who cannot read or write (\%) & + & 0.09 & 0.16 & Census 2005 \\
\hline LoR_03 & Secondary/higher education (\%) & - & 0.13 & N/A & Census 2005 \\
\hline LoR_04 & Travel time to nearest hospital (min.) & + & 0.12 & 0.08 & Municipality \\
\hline LoR_05 & Mean hospital density $\left(\mathrm{km}^{2}\right)$ & - & 0.15 & 0.09 & Municipality \\
\hline LoR_06 & Employed population (\%) & - & 0.11 & 0.12 & Census 2005 \\
\hline LoR_07 & Unemployed population (\%) & + & 0.18 & 0.11 & Census 2005 \\
\hline LoR_08 & People doing housework (\%) & + & 0.11 & 0.13 & Census 2005 \\
\hline \multirow[t]{2}{*}{ LoR_09 } & Retired people (\%) & + & 0.07 & 0.13 & Census 2005 \\
\hline & & & Sum $=1$ & Sum $=1$ & \\
\hline
\end{tabular}

${ }^{a}$ Sign indicates if high indicator values increase (+) or decrease (-) vulnerability.

were subtracted from the normalized values of the expert-based approach $\left(V U_{\text {exp }}\right)$ :

$$
\Delta=V U_{\exp }-V U_{s t a t}
$$

To identify clusters of features with values similar in magnitude, a cluster analysis was performed using Anselin Local Moran's I statistic [52] based on the difference $(\Delta)$ between both approaches.

\section{Results}

Vulnerability indicators

Following a multivariate statistical analysis (i.e., test for multicollinearities and PCA), 15 indicators were retained for statistical modeling. Two additional indicators (i.e., households without water access; secondary/higher education), that were excluded from the statistical modeling approach after multivariate analysis, were kept for the final construction of the expert-based composite vulnerability index as they were identified as relevant by the experts. Table 2 provides an overview of the final set of indicators and lists some of their properties, such as their sign, weights (including expert-based and statistical weights) and data source. Looking at the spatial distribution of the singular raw indicators (Figure 3 ) provides an initial idea how the city is characterized by the different socioeconomic variables.
Socioeconomic vulnerability to dengue fever - comparing modeling approaches

Figure $4 \mathrm{a}$ illustrates the spatial variation in socioeconomic vulnerability to dengue fever based on statistical modeling. In contrast, Figure $4 \mathrm{~b}$ shows the same results, for the expert-based modeling approach, where indicators and weights were selected based on expert opinion. In both maps, neighborhoods of high socioeconomic vulnerability are displayed in red, while neighborhoods with low values are displayed in green.

We conducted a Pearson correlation test among both expert-based and statistical vulnerability scores, indicating a strong positive relationship $(r=0.98)$. A comparison of descriptive statistics, however, has shown that the expert-based approach has a slightly higher mean (0.53) and median (0.56), compared to the purely statistical results $($ mean $=0.48$; median $=0.49$ ).

Both approaches revealed clusters of high levels of socioeconomic vulnerability in the eastern side of the city (comprising communes 13,14, 15, 16 and 21), as well as the north-eastern side (commune 6), and western part of the city (comprising communes 1, 18 and 20). These are poor neighborhoods with high percentages of young (i.e., < 15 years) and illiterate residents, as well as a high proportion of individuals being unemployed or doing housework. Some neighborhoods bordering commune 3 and 9 also revealed higher vulnerability compared to other neighborhoods (Figure 4). These neighborhoods 


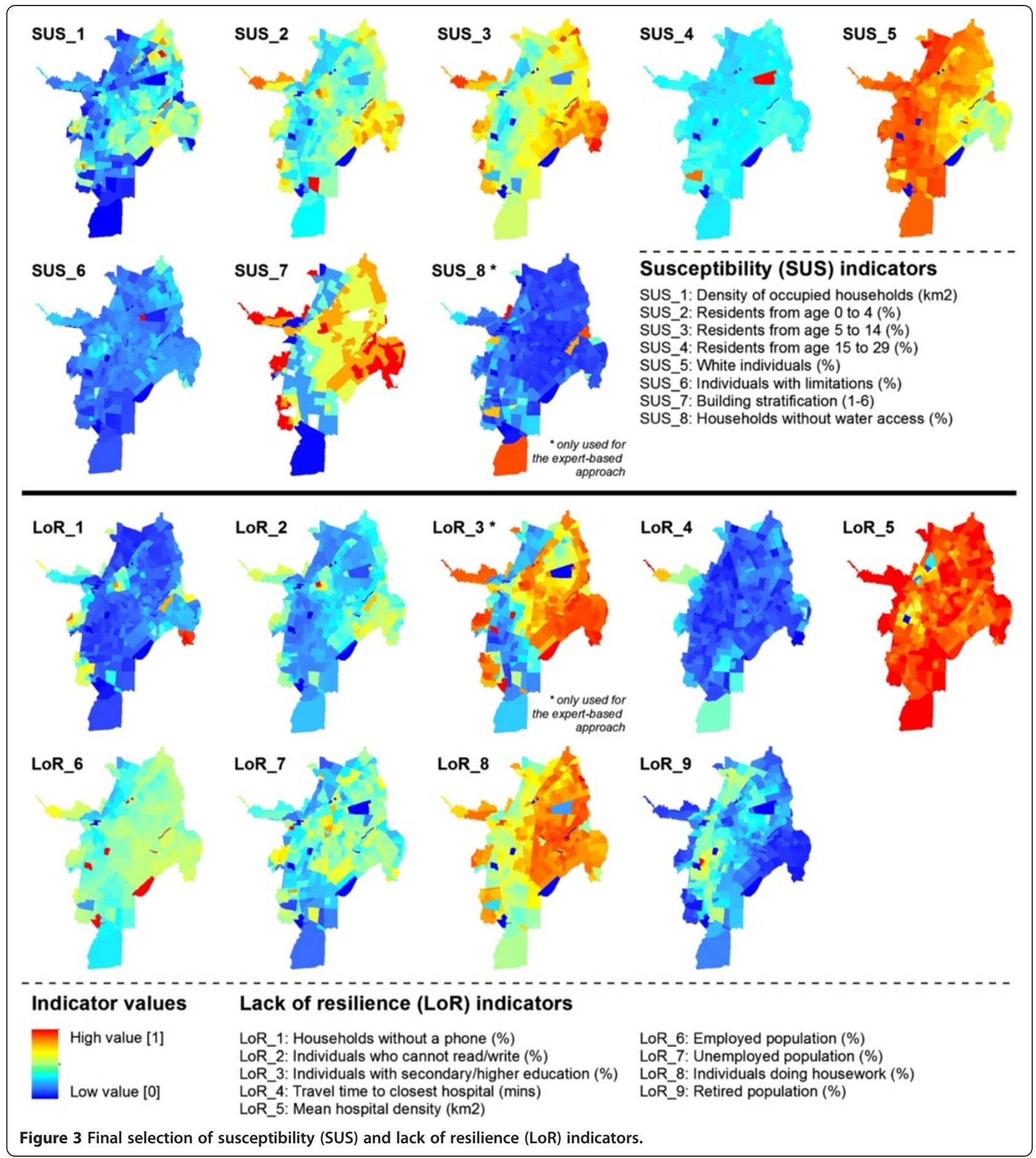

are characterized by high population density and lack of water infrastructure.

The difference between both modeling approaches is displayed in Figure 5.

The model outputs of both approaches have a high correlation, both globally, and spatially. There are, however, also distinct spatial discrepancies between both approaches.
For example, within communes 2, 3, 19 or 21 the expertbased approach has revealed lower levels of vulnerability, while several communes in the eastern part (e.g. communes $8,10,11,12,13,16)$ and southern part (commune 21) have revealed slightly higher vulnerability levels. This pattern can be partly explained by the fact that the expertbased assessment integrated two additional indicators 


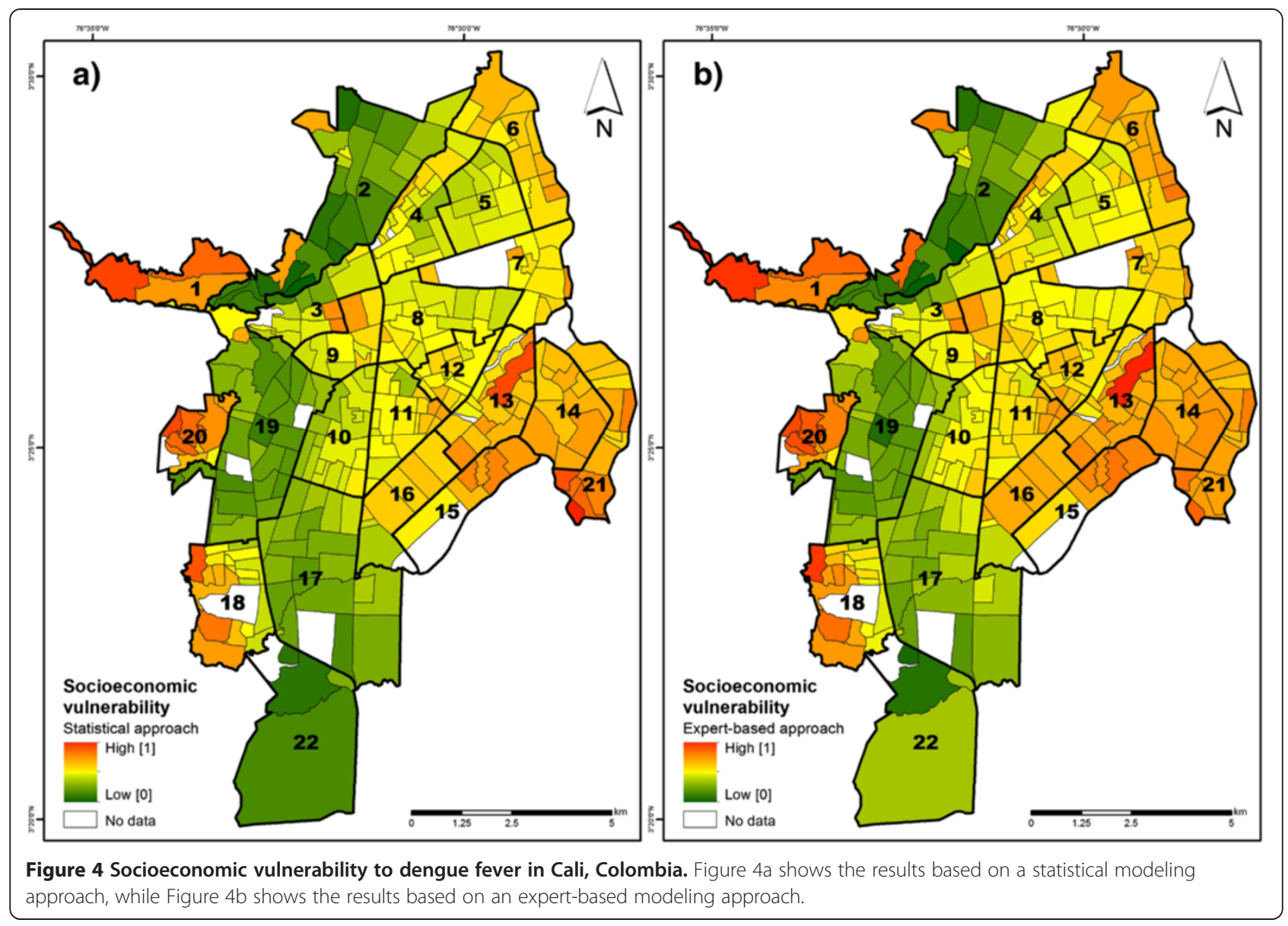

(i.e., households without water access; secondary/higher education) which were removed for the statistical modeling. To highlight clusters of neighborhoods with significant differences, the outcomes of a local clustering method (Local Moran's I) have been added as an additional layer (see Figure 5). HH (i.e., high; high) indicates that a neighborhood where the expert-based approach exhibits higher vulnerability values than the purely statistical approach is surrounded by other neighborhoods characterized by the same pattern. In contrast, LL (i.e., low; low) indicates that a neighborhood where the expert-based approach revealed lower vulnerability values than the purely statistical approach is surrounded by neighborhoods that show a similar pattern. HL (i.e., high; low) represents areas where the expert-based approach revealed higher vulnerability values that are surrounded by areas where the expert-based approach revealed lower vulnerability values than the statistical approach. Figure 5 also indicates that there is no significant local clustering in major parts of the city.

\section{Exploratory tools for visualizing multi-dimensional vulnerability}

As part of any vulnerability study, an interaction with the community and decision makers is highly recommended.
In addition to traditional maps, the outcomes of the analyses were incorporated within an online visualization tool. Current web-based visualization tools offer tremendous capabilities for publishing and disseminating geospatial information to a world-wide audience [53]. We used ArcGIS Explorer Online as an advanced Web-GIS portal to help organize, analyze, and illustratively visualize the results of our study (Figure 6). Such Web-GIS portals provide a collaborative platform for users to explore vulnerabilities towards infectious diseases in general and dengue fever in particular. This dissemination platform is particularly attractive to decision makers who must allocate prevention resources: for example, when selecting a particular neighborhood, a decision maker may retrieve valuable information on the underlying vulnerability indicators, and their relationship to the vulnerability for the entire neighborhood. A nice attribute of this dissemination platform is that it can integrate additional visual outputs such as piecharts, half-donuts, or bar charts.

\section{Discussion}

Reducing the burden of vector-borne diseases without vaccines, such as dengue, requires integrated approaches that take into account both vector or pathogen exposure 


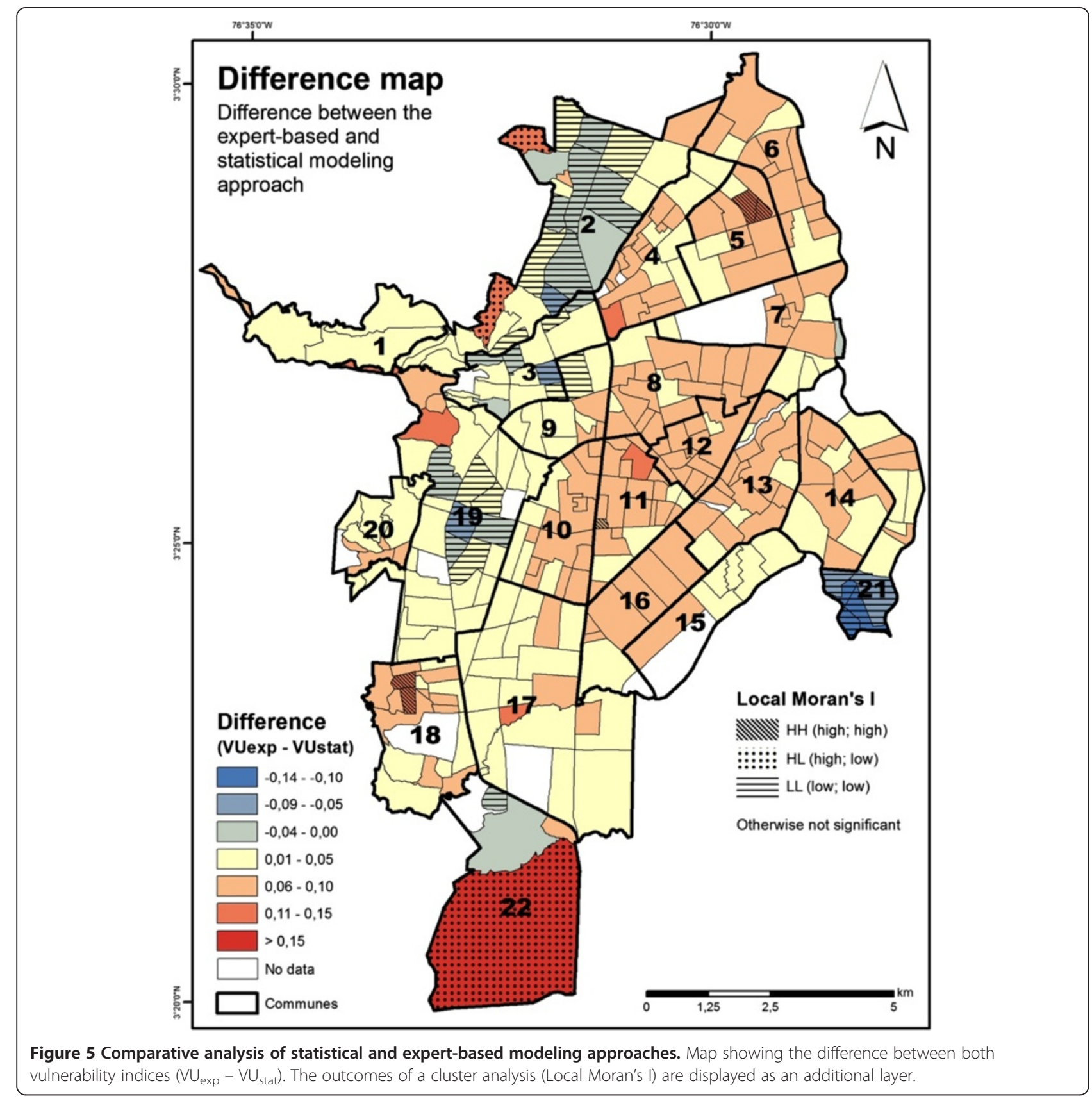

as well as human susceptibility to the disease [31]. Our study aimed at assessing prevailing socioeconomic vulnerabilities to dengue fever independent of the spatial distribution of the hazard (i.e., the disease) based on purely statistical and expert-based approaches taking into account a large set of representative census data and ancillary geospatial datasets; an approach which has not been pursued so far. The assessment was based on a holistic, integrative, yet decomposable, conceptual vulnerability framework. It served as a guidance tool for the establishment of a representative system of indicators, and thus enabled the construction of a composite vulnerability index based on appropriate indicators.

We identified vulnerability hotspots (i.e., clusters of neighborhoods with high levels of vulnerability to dengue fever) across the 340 neighborhoods in Cali, Colombia. By decomposing these hotspots into their underlying socioeconomic vulnerability factors (Figure 6), our approach not only provides information on the neighborhoods where intervention options are most warranted, but also indicates which factors need to be 


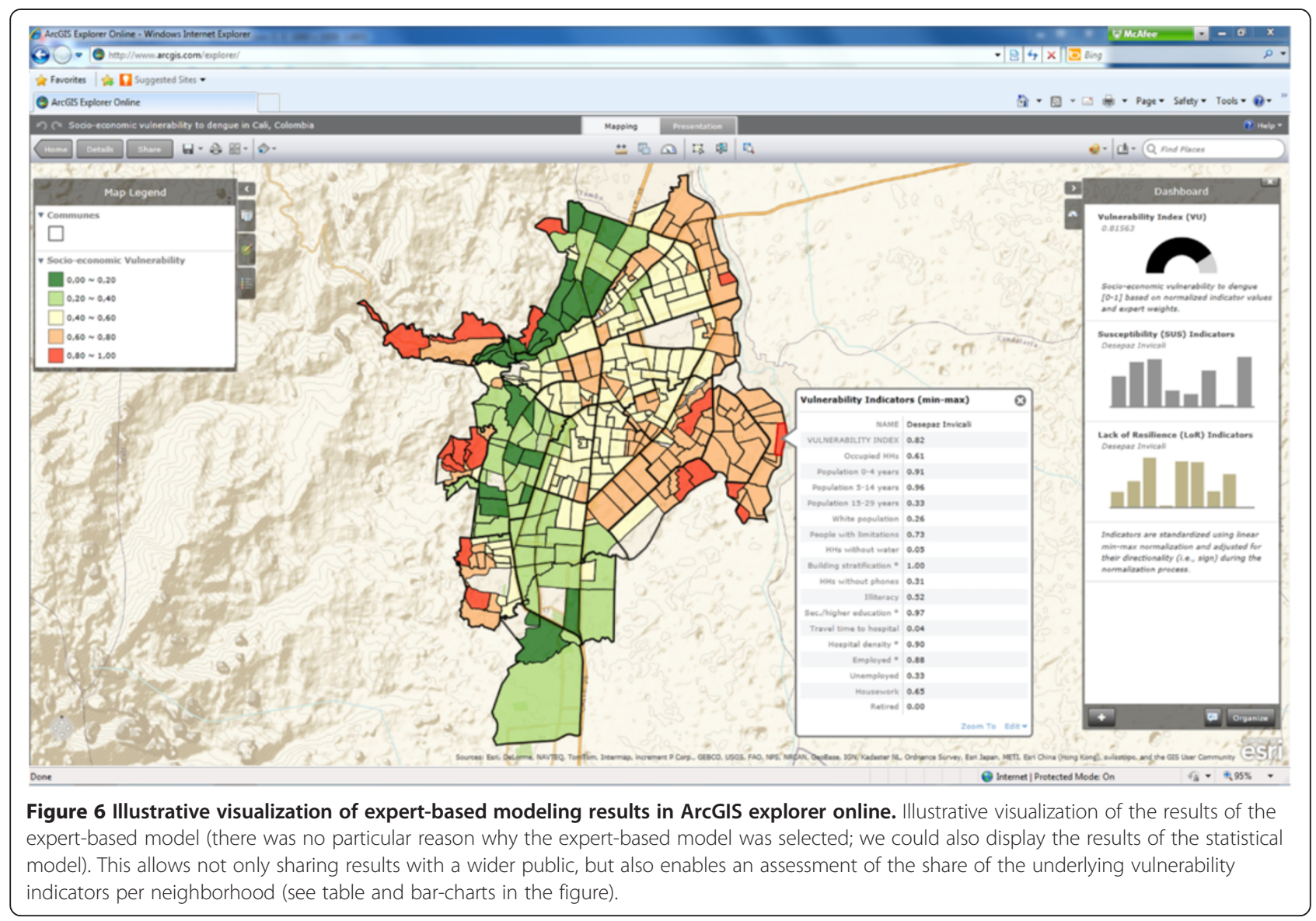

given priority to effectively reduce existing susceptibilities and increase resilience to the disease.

The framework proposed in this paper also comes along with specific challenges. First, the modeling approach is not spatially explicit as it builds on indicators which were reported at the neighborhood level, thus neglecting the 'true' spatial distribution of existing vulnerabilities within the study area. It also results in 'artificial boundaries' that may further lead to aggregation problems as described by Openshaw [54]. Providing a more 'realistic' picture of existing vulnerabilities calls for spatially explicit modeling approaches, which enable a delineation of homogeneous units of vulnerability [55], independent of a-priori defined 'artificial' boundaries. Secondly, additional indicators such as extent and coverage of media campaigns or funding for dengue control programs [31] were not available and thus not integrated into the vulnerability analysis. Third, our approach is not temporally explicit: we considered the census and geospatial datasets at a particular point in time to construct a vulnerability index. Frequent updates and continuous monitoring of prevailing vulnerabilities are particularly needed in rapidly changing urban environments. Finally, one key challenge which warrants further research is the scientific validation of the modeling results. As vulnerability cannot be measured in real world, its validation remains both a hot topic as well as a scientific challenge $[20,31,55]$. However, when integrating the results of the vulnerability assessment in a dengue risk framework, the availability of incidence data (i.e., the manifestation of risk) could help to validate the resulting risk maps. Despite constraints associated with the validation of vulnerability assessments [20,31,55], a kernel density estimator was used to create a surface showing the density of dengue cases (in $\mathrm{km}^{2}$ ) for 2010 (January-December). Both, the spatial distribution of cases and the resulting dengue fever intensity layer, were mapped and the latter was visualized on top of the two vulnerability maps (Figure 7) to enable a visual comparison of vulnerable neighborhoods and areas at risk of contracting the disease (reflected by the dengue intensity surface).

Figure 7 shows that very high densities of dengue cases (i.e., $>200$ cases $/ \mathrm{km}^{2}$ ) tend to concentrate in the western, highly vulnerable fringes of the city, thus placing these areas at high risk. High densities (i.e., $101-200$ cases $/ \mathrm{km}^{2}$ ) 


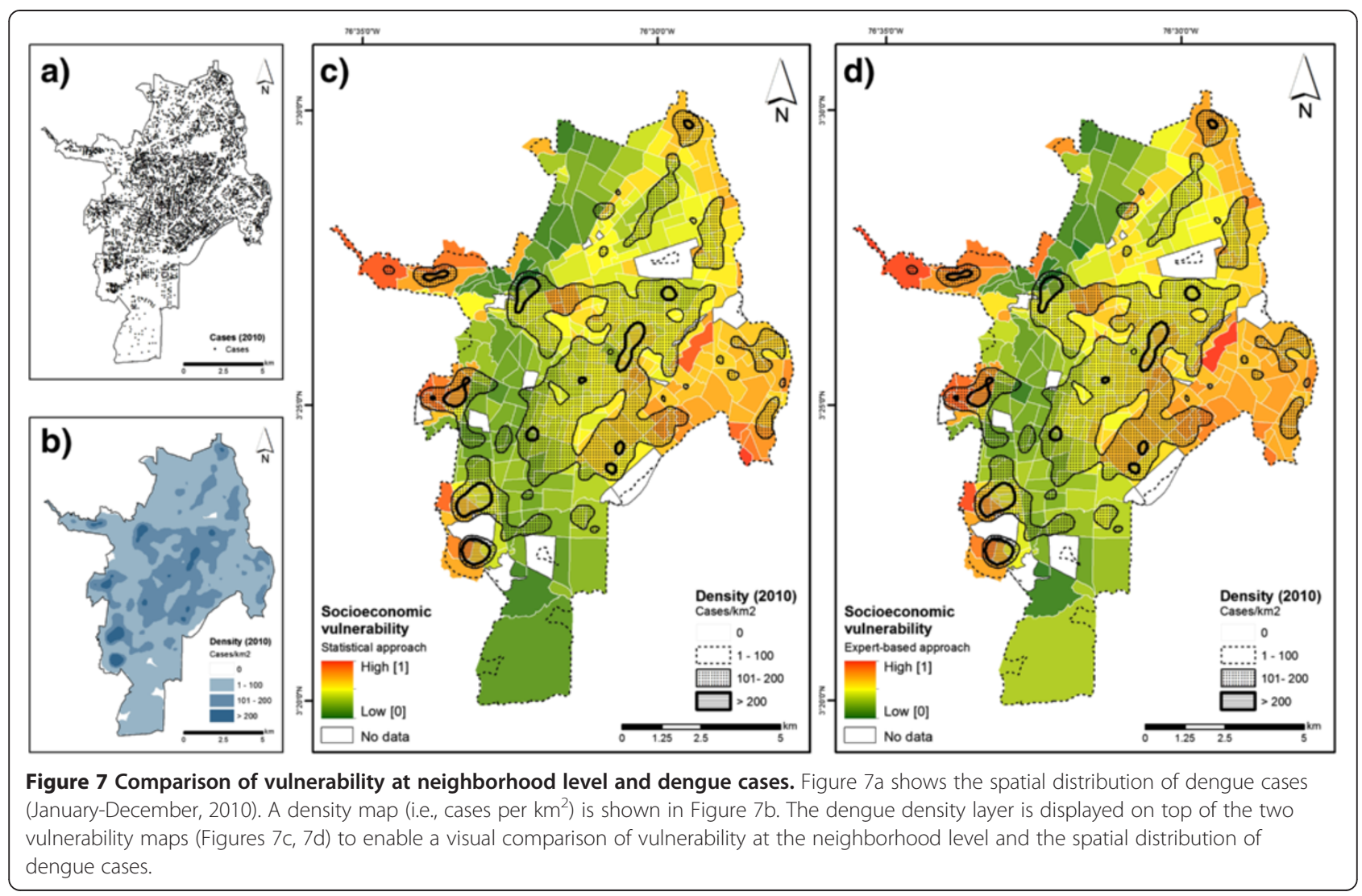

are prevalent in the central and eastern part of the city (areas of medium socioeconomic vulnerability), while lower densities (i.e., $1-100$ cases $/ \mathrm{km}^{2}$ ) are distributed all over the city, also affecting areas of low socioeconomic vulnerability (Figure 7).

To increase user confidence in the applicability of the approach, future work will assess the sensitivity of the approach as well as the validity of the results by further investigating the spatial and statistical relationship between both the modeling results and the individual vulnerability indicators and dengue fever prevalence in the study area.

Findings from this research are particularly salient for public health authorities. First, for the planning of preventive control strategies, for example educational campaigns can be organized with participation from the community, which are likely to increase awareness and proper practices towards the virus. Focusing more resources on particularly vulnerable areas of the city can aid in changing people's awareness and modify people's behavior and attitude towards dengue fever (e.g., use of insecticide treated nets, etc.). It can also assist in planning preventive spraying of bacterial larvicides to permanent stagnant water sources. Second, it can facilitate timely response strategies during an outbreak by pointing towards areas that are more vulnerable to be controlled first, with the objective of minimizing the impact of the virus.

\section{Conclusions}

Statistical and expert-based approaches were utilized for the modeling of prevailing vulnerabilities to dengue in the urban tropical environment of Cali, Colombia independent of the current distribution of the disease. Using neighborhoods as the spatial reporting unit, we integrated various socioeconomic and demographic indicators derived from census data and ancillary datasets into a final composite vulnerability index. A conceptual framework was used as a guidance tool for the development of a representative set of vulnerability indicators, thus enabling the reproducibility and comparability of modeling results. The methods presented in this paper make an important contribution as a decision support tool for reducing existing vulnerabilities and strengthening or building up resilience to vector-borne diseases in general, and dengue in particular. The results of our analysis also provide relevant information for decision makers in Cali, Colombia, as they not only help prioritizing intervention areas (i.e., vulnerability hotspots), but also indicate which factors need to be addressed foremost in the context of targeted intervention measures. 


\section{Competing interests}

The authors declare that they have no competing interests.

\section{Authors' contributions}

$\mathrm{MH}$ and SK were responsible for the conceptualization and design of the study. IC and ED were responsible for data collection and pre-processing. $\mathrm{MH}$ was responsible for data analysis, interpretation of results, and for drafting the manuscript (incl. the figures). ED, IC and SK provided substantial input to the manuscript and the interpretation of modeling results. All authors read and approved the final manuscript.

\section{Acknowledgements}

We would like to thank MetroCali for the city's street network and bus routes, the panel of experts for their input, and the Public Health Secretariat of the city of Cali for their support. The research leading to these results has received funding from the European Union's Seventh Framework Programme (FP7/2007-2013) under grant agreement no. 266327 (HEALTHY FUTURES, http://www.healthyfutures.eu/) and from the Austrian Science Fund (FWF) through the Doctoral College GIScience (DK W 1237-N23).

\section{Author details}

'Interfaculty Department of Geoinformatics - Z_GIS, University of Salzburg, Schillerstraße 30, Salzburg 5020, Austria. ${ }^{2}$ Department of Geography and Earth Sciences, University of North Carolina at Charlotte, Charlotte, NC 28223, USA. ${ }^{3}$ Department of Social Sciences, Louisiana Tech University, Ruston, LA 71272, USA.

Received: 5 June 2013 Accepted: 8 August 2013

Published: 14 August 2013

\section{References}

1. Githeko AK, Lindsay SW, Confalonieri UE, Patz JA: Climate change and vector-borne diseases: a regional analysis. Bull World Health Organ 2000, 78(9):1136-1147.

2. Gubler DJ: Resurgent vector-borne diseases as a global health problem. Emerg Infect Dis 1998, 4(3):442-450.

3. Sutherst RW: Global change and human vulnerability to vector-borne diseases. Clin Microbiol Rev 2004, 17(1):136-173.

4. Intergovernmental Panel on Climate Change: Climate change 2007: the physical science basis. contribution of working group I to the fourth assessment report of the intergovernmental panel on climate change. Cambridge, UK, and New York, NY, USA: Cambridge University Press; 2007.

5. Gubler DJ: Epidemic dengue/dengue hemorrhagic fever as a public health, social and economic problem in the 21 st century. Trends Microbiol 2002, 10:100-103.

6. Sachs J, Malaney P: The economic and social burden of malaria. Nature 2002, 415:680-685.

7. Bhatt S, Gething PW, Brady OJ, Messina JP, Farlow AW, Moyes CL, Drake JM, Brownstein JS, Hoen AG, Sankoh O, Myers MF, George DB, Jaenisch T, Wint GRW, Simmons CP, Scott TW, Farrar JJ, Hay SI: The global distribution and burden of dengue. Nature 2013, 496(7446):504-507.

8. World Health Organization: Dengue and severe dengue. Factsheet $N^{\circ} 117$. 2012. http://www.who.int/mediacentre/factsheets/fs117/en/index.html.

9. Simmons CP, Farrar JJ, Nguyen VV, Wills B: Dengue. N Engl J Med 2012, 366(15):1423-1432.

10. Braga C, Luna CF, Martelli CM, de Souza WW, Cordeiro MT, Alexander N, de Albuquerque MF, Júnior JC, Marques ET: Seroprevalence and risk factors for dengue infection in socio-economically distinct areas of Recife Brazil. Acta Trop 2010, 113(3):234-240.

11. Getis A, Morrison AC, Gray K, Scott TW: Characteristics of the spatial pattern of the dengue vector, Aedes aegypti, in Iquitos, Peru. Am J Trop Med Hyg 2003, 69(5):494-505.

12. Honório NA, Nogueira RMR, Codeço CT, Carvalho MS, Cruz OG, Magalhães MDAFM, De Araújo JMG, de Araújo ESM, Gomes MQ, Pinheiro LS, da Silva Pinel C, Lourenço-de-Oliveira R: Spatial evaluation and modeling of Dengue seroprevalence and vector density in Rio de Janeiro Brazil. PLoS Negl Trop Dis 2009, 3(11):1-11.

13. Paz-Soldan VA, Plasai V, Morrison AC, Rios-Lopez EJ, Guedez-Gonzales S, Grieco JP, Mundal K, Chareonviriyaphap T, Achee NL: Initial assessment of the acceptability of a push-pull aedes aegypti control strategy in lquitos, Peru and Kanchanaburi Thailand. Am J Trop Med Hyg 2011, 84(2):208-217.
14. Teixeira M, Barreto ML, Costa Mda C, Ferreira LD, Vasconcelos PF, Cairncross S: Dynamics of dengue virus circulation: a silent epidemic in a complex urban area. Trop Med Int Health 2002, 7(9):757-762.

15. Troyo A, Fuller DO, Calderón-Arguedas O, Solano ME, Beier JC: Urban structure and dengue fever in Puntarenas, Costa Rica. Singap J Trop Geogr 2009, 30(2):265-282.

16. Romero-Vivas CM, Leake CJ, Falconar AK: Determination of dengue virus serotypes in individual Aedes aegypti mosquitoes in Colombia. Med Vet Entomol 1998, 12:284-288.

17. Mendez JA, Usme-Ciro JA, Domingo C, Rey GJ, Sanchez JA, Tenorio A, Gallego-Gomez JC: Phylogenetic history demonstrates two different lineages of dengue type 1 virus in Colombia. Virol J 2010, 7(226):1-12.

18. Cali, SdSPMd: Historia del dengue en Cali. Endemia o una continua epidemia. Cali: Secretaria de Salud Publica Municipal de Cali; 2010.

19. Delmelle E, Casas I, Rojas J, Varelo A: Modeling spatio-temporal patterns of dengue fever, Colombia. Int J Applied Geospatial Research. in press.

20. Birkmann J: Measuring Vulnerability to Towards Disaster Resilient Societies. Shibuya-ku, Tokyo, Japan: Nations University Press; 2006.

21. Cutter SL, Boruff BJ, Shirley WL: Social vulnerability to environmental hazards. Soc Sci Quart 2003, 84:242-261.

22. Füssel HM: Vulnerability: a generally applicable conceptual framework for climate change research. Glob Environ Change 2007, 17(2):155-167.

23. Intergovernmental Panel on Climate Change: Managing the risks of extreme events and disasters to advance climate change adaptation. a special report of working groups I and II of the intergovernmental panel on climate change. Cambridge, UK, and New York, NY, USA: Cambridge University Press; 2012:582.

24. Bates I, Fenton C, Gruber J, Lalloo D, Lara AM, Squire SB, Theobald S, Thomson R, Tolhurst R: Vulnerability to malaria, tuberculosis, and HIV/ AIDS infection and disease. Part 1: determinants operating at individual and household level. Lancet Infect Dis 2004, 4:267-277.

25. Bates I, Fenton C, Gruber J, Lalloo D, Lara AM, Squire SB, Theobald S, Thomson R, Tolhurst R: Vulnerability to malaria, tuberculosis, and HIV/ AIDS infection and disease. Part II: determinants operating at environmental and institutional level. Lancet Infect Dis 2004, 4:368-375.

26. Semenza JC, Suk JE, Estevez V, Ebi KL, Lindgren E: Mapping climate change vulnerabilities to infectious diseases in Europe. Environ Health Perspect 2012, 120(3):385-392.

27. De Mattos Almeida MC, Caiaffa WT, Assuncao RM, Proietti FA: Spatial vulnerability to dengue in a Brazilian urban area during a 7-year surveillance. J Urban Health 2007, 84(3):334-345.

28. Martinez TTP, Rojas LI, Valdés LS, Noa RR: Spatial vulnerability to dengue: an application of the geographic information systems in Playa municipality, City of Havana. Revista Cubana de Salud Pública 2003, 29(4):353-365.

29. Mazrura S, Rozita H, Hidayatulfathi O, Zainudin MA, Mohamad Naim MR, Nadia Atiqah MN, Rafeah MN, Er AC, Norela S, Nurul Ashikin Z, Joy JP: Community vulnerability on dengue and its association with climate variability in Malaysia: a public health approach. Malaysian Journal of Public Health Medicine 2010, 10(2):25-34.

30. Tipayamongkholgul M, Lisakulruk S: Socio-geographical factors in vulnerability to dengue in Thai villages: a spatial regression analysis. Geospat Health 2011, 5(2):191-198.

31. Dickin SK, Schuster-Wallace CJ, Elliott SJ: Developing a vulnerability mapping methodology: applying the water-associated disease index to dengue in Malaysia. PLOS ONE 2013, 8(5):e63584.

32. Chang AY, Parrales ME, Jimenez J, Sobieszczyk ME, Hammer SC, Copenhaver DJ, Kulkarni RP: Combining Google earth and GIS mapping technologies in a dengue surveillance system for developing countries. Int J Health Geogr 2009, 8:49.

33. Vazquez-Prokopec GM, Stoddard ST, Paz-Soldan V, Morrison AC, Elder JP, Kochel TJ, Scott TW, Kitron U: Usefulness of commercially available GPS data-loggers for tracking human movement and exposure to dengue virus. Int J Health Geogr 2009, 8:68.

34. Vanwambeke SO, van Benthem BHB, Khantikul N, Burghoorn-Maas C, Panart K, Oskam L, Lambin EF, Pradya: Multi-level analyses of spatial and temporal determinants for dengue infection. Int J Health Geogr 2006, 5:5.

35. Stratton LM, O'Neill S, Kruk ME, Bell ML: The persistence of malaria: addressing the fundamental causes of a global killer. Soc Sci Med 2008, 67:854-862.

36. Jones CO, Williams HA: The social burden of malaria: what are we measuring? Am J Trop Med Hyg 2004, 71(2):156-161. 
37. Cali, AdSd: Cali en cifras. Cali: Alcaldia de Cali. 2008. http://planeacion.cali. gov.co/Publicaciones/Cali_en_cifras/Caliencifras2010.pdf.

38. Restrepo LDE: El plan piloto de cali 1950. Revista Bitacora Urbano Territorial 2006, 1:222-223.

39. Casas I, Delmelle E, Varela A: A space-time approach to diffusion of health service provision information. Int Reg Sci Rev 2010, 33(2):134-156.

40. Birkmann J, Cardonna OD, Carreno ML, Barbat AH, Pelling M, Schneiderbauer S, Kienberger S, Keiler M, Alexander D, Zeil P, Welle T: Framing vulnerability, risk and societal responses: the MOVE framework. Nat Hazards 2013. online.

41. Organisation for Economic Co-operation and Development: Handbook on constructing composite indicators: methodology and user guide. 2008. http:// www.oecd.org/std/42495745.pdf.

42. Malczewski J: GIS and multicriteria decision analysis. New York, NY, USA: Wiley; 1999.

43. Guha-Sapir D, Schimmer B: Dengue fever: new paradigms for a changing epidemiology. Emerg Themes Epidemiol 2005, 2:7622-7632

44. Were F: The dengue situation in Africa. Paediatr Int Child Health 2012, 32(1):18-21.

45. Sierra $C B$, Kouri G, Guzman MG: Race: a risk factor for dengue hemorrhagic fever. Arch Virol 2007, 152:533-542.

46. Kouri GP, Guzman MG, Bravo JR, Triana C: Dengue haemorrhagic fever/ dengue shock syndrome: lessons from the Cuban epidemic. Bull World Health 1981, 67:375-380.

47. Moldan B, Dahl AL: Challenges to sustainability indicators. In Sustainability Indicators. A Scientific Assessment. Edited by Hak T, Moldan B, Dahl AL. Washington, DC, USA: Island Press; 2007:1-26.

48. Delmelle EC, Casas I: Evaluating the spatial equity of bus rapid transitbased accessibility patterns in a developing country: the case of Cali, Colombia. Transport Policy 2012, 20:36-46.

49. Wisner B, Blaikie P, Cannon T, Davis I: At risk: natural hazards, people's vulnerability and disasters. New York, NY, USA: Routledge; 2004

50. Rygel L, O'Sullivan D, Yarnal B: A method for constructing a social vulnerability index: an application to hurricane storm surges in a developed country. Mitig Adapt Strat Glob Chang 2006, 11:741-764.

51. Groeneveld RA, Meeden G: Measuring Skewness and Kurtosis. J R Stat Soc Discipline 1984, 33(4):391-399.

52. Anselin L: Local indicators of spatial association-LISA. Geographical analysis 1995, 27(2):93-115.

53. Blaschke T, Donert K, Gossette F, Kienberger S, Marani M, Qureshi S, Tiede D: Virtual globes: serving science and society. Information 2012, 3(3):372-390.

54. Openshaw S: The modifiable areal unit problem. Norwich: Concepts and Techniques in Modern Geography; 1984.

55. Kienberger S, Lang S, Zeil P: Spatial vulnerability units - expert-based spatial modeling of socio-economic vulnerability in the Salzach catchment, Austria. Nat Hazard Earth Sys 2009, 9:767-778.

doi:10.1186/1476-072X-12-36

Cite this article as: Hagenlocher et al:: Assessing socioeconomic vulnerability to dengue fever in Cali, Colombia: statistical vs expertbased modeling. International Journal of Health Geographics 2013 12:36.

\section{Submit your next manuscript to BioMed Central and take full advantage of:}

- Convenient online submission

- Thorough peer review

- No space constraints or color figure charges

- Immediate publication on acceptance

- Inclusion in PubMed, CAS, Scopus and Google Scholar

- Research which is freely available for redistribution

Submit your manuscript at www.biomedcentral.com/submit 